Revista de Investigación

Agraria y Ambiental

Volumen 8 Número 1 Enero - Junio de 2017 ISSN 2145-6097 


\title{
Revista de Investigación Agraria y Ambiental
}

\author{
Volumen 8 Número 1 - enero - junio 2017 - ISSN 2145-6097
}

Cuerpo directivo

JAIME ALBERTO LEAL AFANADOR

Rector UNAD

CONSTANZA ABADÍA GARCíA

Vicerrector Académica y de Investigación

LEONARDO YUNDA PERLAZA

Vicerrector de Medios y Mediaciones Pedagógicas

LEONARDO EVEMELETH SANCHEZ TORRES

Vicerrector de Desarrollo Regional

y Proyección Comunitaria

EDGAR GUILLERMO RODRÍGUEZ

Vicerrector de Servicios a Aspirantes,

Estudiantes y Egresados

LUIGI HUMBERTO LÓPEZ GUZMÁN

Vicerrector de Relaciones Internacionales

JULIALBA ÁNGEL OSORIO

Decana Escuela de Ciencias Agrícolas,

Pecuarias y del Medio Ambiente

JENNY FABIOLA HERNÁNDEZ

Líder Nacional de Investigación UNAD

\section{YOLVI PRADA}

Líder Nacional de Investigación Escuela de Ciencias

Agrícolas, Pecuarias y del Medio Ambiente

\section{REINALDO GIRALDO DIAZ \\ Director Editor}

\section{LIBIA ESPERANZA NIETO GÓMEZ \\ Coeditora}

\section{Comité Editorial}

FLÁVIO VIEIRA MEIRELLES

Médico Veterinario, Ph.d.

Universidad de São Paulo

\section{BEATRIZ EUGENIA CID AGUAYO}

Socióloga, M.sc. y Ph.D. en Sociología

Universidad de Concepción, Chile

OSCAR EMERSON ZUÑIGA MOSQUERA

Ingeniero Agrónomo, Mestro Em Andamento

Em Desenvolvimento e Meio Ambiente

Universidade Federal de Pernambuco Ufpe, Brasil

\section{OSCAR EDUARDO SANCLEMENTE REYES}

Ingeniero Ambiental, M.sc., Ph.D. en Agroecología

Universidad Nacional de Colombia
ROLANDO TITO BACCA IBARRA

Ingeniero Agrónomo, M.sc., Ph.D. en Entomología

Universidad de Nariño

\section{Comité Científico}

\section{HERNÁN JAIR ANDRADE CASTAÑEDA}

Ingeniero Agrónomo, M.sc., Ph.d.

Universidad del Tolima

\section{ALVEIRO SALAMANCA JIMÉNEZ \\ Ingeniero Agrónomo, Ph.D. \\ University Of California, Davis}

\section{ELISABETE FIGUEIREDO}

Socióloga, Ph.D. en Environmental Sciences

University of Aveiro

Revisor de Estilo Lengua Inglesa

WILLIAM FRANCIS

B.T.A. - OREGON

\section{Revisor de Estilo Lengua Portuguesa}

\section{SAMUEL DIOGO MEIRINHO}

Universidade de Aveiro - Portugal

\section{Revisor de Estilo Lengua Española}

\section{EFIGENIO HERNÁNDEZ}

Universidad Nacional Abierta y a Distancia

\section{Fotografía de la Portada}

Cosecha de café, Finca El Guamo,

El Tambo. Cauca. Colombia.

Tomada y cedida por: Martha Isabel Cabrera Otálora

\author{
IMPRESIÓN \\ PICTOGRAMA S.A.S.
}

INFORMACIÓN, CORRESPONDENCIA, SUSCRIPCIONES Y CANJE REVISTA DE INVESTIGACIÓN AGRARIA Y AMBIENTAL

Escuela de Ciencias Agrícolas, Pecuarias y del Medio Ambiente Universidad Nacional Abierta y a Distancia

Calle 14 Sur N. 14-23 Bogotá, Colombia

Teléfonos: (571) 3443700 ext. 1529

e-mail: riaa@unad.edu.co

La revista puede consultarse en su versión electrónica en: http://hemeroteca.unad.edu.co/index.php/riaa/index 


\section{Presentación}

La Revista de Investigación Agraria y Ambiental (RIAA) es un proyecto editorial de la Universidad Nacional Abierta y a Distancia (UNAD), que surge en el año 2009 con el fin de comunicar los resultados de investigaciones originales en el área agraria y ambiental realizadas por personas, grupos o instituciones tanto nacionales como internacionales. Con el fin de mantener y afianzar la confianza entre investigadores y público interesado en las temáticas de RIAA, la revista busca su inclusión en prestigiosas bases de datos y sistemas de indexación tanto nacionales como internacionales.

\section{Misión}

La misión de RIAA es fomentar la comunicación y colaboración entre investigadores nacionales e internacionales a través de la divulgación y transferencia de conocimiento relacionado con las ciencias agrarias y del medio ambiente, con el fin de fortalecer la generación de nuevo conocimiento.

\section{Público al que se dirige}

La Revista de Investigación Agraria y Ambiental (RIAA) es una publicación oficial de la Escuela de Ciencias Agrícolas, Pecuarias y del Medio Ambiente (ECAPMA) de la Universidad Nacional Abierta y a Distancia (UNAD), destinada a publicar artículos resultantes de las investigaciones originales en el área agraria y ambiental y en las áreas de conocimiento afines, en temas relacionados con los avances en producción animal, agricultura y uso sostenible de los recursos naturales. Como ejemplos de áreas afines citamos, entre otras, ética, ecología, sociología, geografía, historia, derecho, educación y economía, cuando se ocupan de perspectivas del desarrollo, de estilos de agricultura, de historia agraria, de desarrollo sustentable, de bioética y ética ambiental, de educación ambiental y extensión rural, de política agraria y ambiental, de legislación ambiental, forestal y agraria o de contribuciones significativas e innovadoras con visión sistémica, interdisciplinaria y/o transdisciplinaria. La publicación circula en formato impreso y en forma electrónica con acceso libre.

\section{Periodicidad}

RIAA es una publicación semestral (enero-junio y julio-diciembre). 


\section{Cesión de derechos}

RIAA, al momento de recibir la postulación de un manuscrito por parte de su autor, ya sea a través de correo electrónico o postal, considera que puede publicarse en formatos físicos y/o electrónicos y facilitar su inclusión en bases de datos, hemerotecas y demás procesos de indexación.

Se autoriza la reproducción y citación del material de la revista, siempre y cuando se indique de manera explícita el nombre de la revista, los autores, el título del artículo, volumen, número y páginas.

Las ideas y conceptos expresados en los artículos son responsabilidad de los autores y en ningún caso reflejan las políticas institucionales de la UNAD. 


\section{Indexaciones}

La Revista de Investigación Agraria y Ambiental es indexada en las siguientes bases de datos especializadas.

\section{Publindex

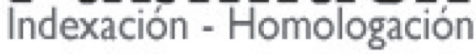

La Base Bibliográfica Nacional - BBN Publindex, que hace parte del Sistema Nacional de Indexación y Homologación, es dirigida por el Departamento Administrativo de Ciencia, Tecnología e Innovación, Colciencias. Está constituida por la información integrada por las revistas especializadas de CT+l sobre su producción, donde se hace visible para consulta en línea la información bibliográfica recolectada de los documentos hasta el nivel de resumen.
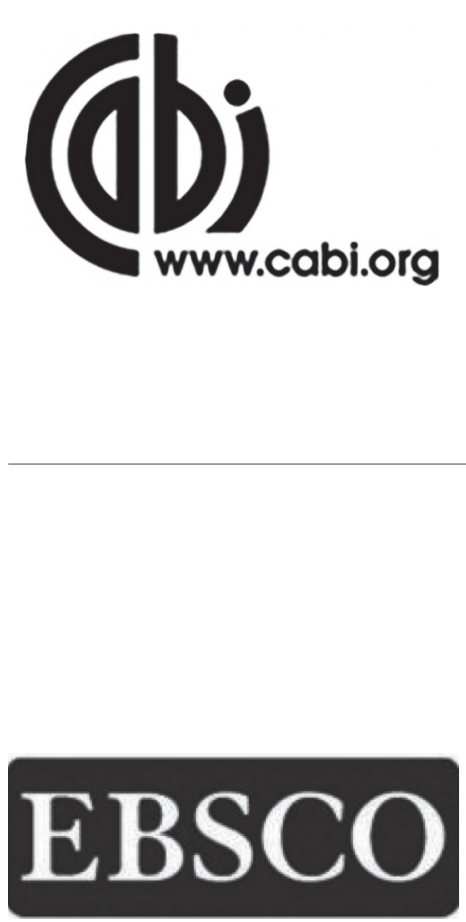

CABI es una organización intergubernamental sin fines de lucro, que proporciona información y servicios de carácter científico en el mundo desarrollado y en desarrollo.

CAB Abstracts: es una base de datos que ayuda a documentar la literatura publicada en el mundo, en: agricultura, medio ambiente, ciencias veterinarias, ciencias vegetales, micología y parasitología, economía aplicada, ciencias de la alimentación, salud humana, nutrición y temas relacionados.

Repositorio de Texto Completo de CABI: garantiza que los artículos estén disponibles y sean fácilmente localizados por científicos y profesionales a nivel mundial.
EBSCO ofrece un repositorio de: documentos, audio libros, libros digitales y bases de datos que cubren diferentes áreas, niveles de investigación e instituciones: escuelas, bibliotecas públicas, universidades, entidades de salud, corporaciones y agencias gubernamentales.

ENVIRONMENT INDEX: Base de datos especializada; información en temáticas relacionadas con: agricultura, ciencias del mar y agua dulce, ecología de ecosistemas, geografía, energía, fuentes renovables de energía, recursos naturales, contaminación y gestión de residuos, tecnología ambiental, legislación ambiental, políticas públicas, planificación urbana e impactos sociales.

FUENTE ACADEMICA PREMIER: Esta base de datos proporciona una colección de revistas científicas de América Latina, Portugal y España; cubre todas las áreas temáticas con especial énfasis en agricultura, ciencias biológicas, economía, historia, derecho, literatura, filosofía, psicología, administración pública, religión y sociología. 


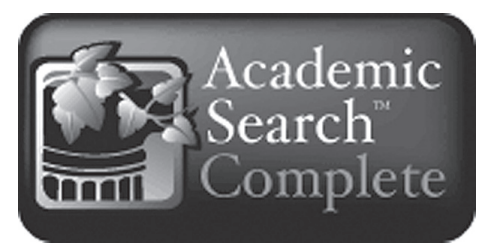

ACADEMIC SEARCH COMPLETE ofrece una gran colección de revistas académicas multidisciplinarias en texto completo, proporcionando a los usuarios acceso a información crítica de muchas fuentes únicas. Además, incluye texto completo revisado por pares. El contenido académico cubre una amplia gama de importantes áreas incluyendo antropología, ingeniería, derecho, ciencias y muchas más.

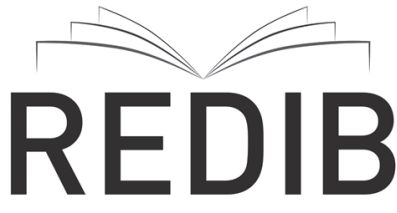

Red Iberoamericana de Innovación y Conocimiento Científico
REDIB (RED IBEROAMERICANA DE INNOVACIÓN Y CONOCIMIENTO CIENTÍFICO) es una plataforma para la agregación de contenidos científicos y académicos en formato electrónico en el espacio lberoamericano. REDIB proporciona acceso y facilita la difusión y ejecución del trabajo científico. Los destinatarios de esta información son tanto la comunidad académica y sociedad en general, así como los responsables de la política científica y aquellos encargados de ponerla en práctica y analizarla.

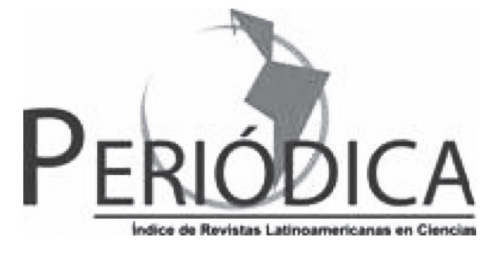

O Dialnet
PERIÓDICA es una base de datos bibliográfica creada en 1978 en la Universidad Nacional Autónoma de México (UNAM). La base de datos se actualiza diariamente y más de 10 mil registros son agregados cada año.

Ofrece alrededor de 336 mil registros bibliográficos de artículos originales, informes técnicos, estudios de caso, estadísticas y otros documentos publicados en cerca de 1500 revistas de América Latina y el Caribe, especializadas en ciencia y tecnología.

Dialnet es una base de datos de acceso libre, creada por la Universidad de La Rioja (España), que difunde producción científica hispana. 


\section{Proquest}

ProQuest conserva amplia y variada información, tanto de archivos históricos, como de los avances científicos actuales y maneja tecnologías digitales que optimizan la búsqueda, intercambio y gestión de la información. ProQuest proporciona servicios en el ámbito académico, empresarial, gubernamental, bibliotecas escolares y públicas, así como servicios a los investigadores profesionales, que les permiten la adquisición estratégica, gestión y búsqueda de colecciones de información.

Actualmente RIAA es visible en las bases de datos: ProQuest Agricultultural Science Collection, ProQuest SciTech Collection y ProQuest Natural Science Collection.

ProQuest Agricultultural Science Collection: Proporciona una amplia cobertura de todos los temas de agricultura y es apoyada por la Biblioteca Agrícola Nacional de EE.UU. La interfaz aporta características avanzadas y herramientas que permiten a los investigadores más precisión en las revisiones de literatura especializada y adaptada a su área del estudio.

ProQuest SciTech Collection: Combina una serie de bases de datos especializadas en Ciencias Naturales, Tecnología e Ingeniería en una interfaz dinámica que permite acceso a texto completo mediante una búsqueda integral a través de amplios resúmenes gestionados por equipos editoriales de expertos.

ProQuest Natural Science Collection: Proporciona una vasta cobertura de literatura en el ámbito de: Agricultura, Biología, Geología, Ciencias de la Tierra y Ciencias Ambientales. Ofrece acceso a texto completo de fuentes que incluyen: publicaciones académicas, revistas profesionales, informes, libros, actas de conferencias y material de relevancia.

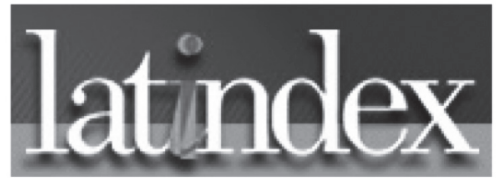

Latindex es un sistema de Información sobre las revistas de investigación científica, técnico-profesionales y de divulgación científica y cultural que se editan en los países de América Latina, el Caribe, España y Portugal. La idea de creación de Latindex surgió en 1995 en la Universidad Nacional Autónoma de México (UNAM) y se convirtió en una red de cooperación regional a partir de 1997.

\section{e-revist@s}

La Plataforma Open Access de Revistas Científicas Electrónicas Españolas y Latinoamericanas e-Revistas, es un proyecto impulsado por el Consejo Superior de Investigaciones Científicas (CSIC) con el fin de contribuir a la difusión y visibilidad de las revistas científicas publicadas en América Latina, Caribe, España y Portugal. 


\section{Editorial}

\section{ARTÍCULOS DE INVESTIGACIÓN}

Determinación del riesgo a la erosión potencial hídrica en la zona cafetera del Quindío, Colombia

Determination of the risk to the potential erosion by water in the coffee zone of the Quindio, Colombia

A avaliação de risco para o potencial de erosão hídrica na região do café de Quindio, Colombia

Estimation of the transfer of vascular epiphytes, as a conservation strategy in the municipality of Aguazul, Casanare, Colombia.

Valoración de la transferencia de epífitas vasculares, como una estrategia de conservación en el municipio de Aguazul, Casanare, Colombia

Estimativa da transferência de epífitas vasculares, como estratégia de conservação no município de Aguazul, Casanare, Colômbia.

Liberación ruminal de cuatro fuentes inorgánicas de magnesio por medio de la técnica in situ en ganado Holstein en trópico alto

Release ruminal of four inorganic sources of magnesium through the technique in situ in Holstein cattle in high tropic

Libertação no rúmen de quatro fontes inorgânicas de magnésio pela técnica in situ em Holsteins elevados trópicos

Cambios en la orientación productiva en el Garbanzo, Irapuato, Guanajuato, México. ¿De chiveros a porcicultores?

Changes in the productive orientation in Garbanzao, Irapuato, Guanajuato, Mexico. From goat producers to pig producers?

Mudanças na orientação produtiva em Garbanzo, Irapuato, Guanajuato, México. ¿De produtores de cabros a produtores de suínos? 
Isolamento de lectinas por cromatografia de afinidade

Aislamiento de lectinas por cromatografía de afinidad

Isolation of lectins by affinity chromatography

José Camilo Torres Romero, Myriam Janeth Ortega Torres, Jhon Alexander

Infante Betancour, Cicero Antonio Maia Cavalcante

Bioprospección de hongos micorrízicos arbusculares como alternativa para el fortalecimiento del cultivo de aguacate (Persea americana Miller) en Colombia

Bioprospecting of fungi mycorrhizal mycorrhizal as an alternative for the strengthening of the cultivation of avocado (Persea americana Miller) in Colombia

Bioprospecção fungos micorrízicos arbusculares como uma alternativa para fortalecer o cultivo de abacate (Persea americana Miller) na Colômbia

Sandra Patricia Montenegro Gómez, Silvia Eugenia Barrera Berdugo, Carlos Mario Valencia

Friabilidad del suelo: métodos de estimación con énfasis en la determinación cuantitativa de la resistencia al rompimiento

Soil friability: estimation methods with emphasis on the quantitative determination of breaking resistance

Friabilidade do solo: métodos de estimação com um ênfase na

determinação quantitativa da força de ruptura

Edgar Alvaro Avila Pedraza

Marco teórico para la construcción de una propuesta de turismo rural comunitario

Theoretical framework for the construction of a proposal of rural community tourism

Quadro teórico para a construção de uma proposta para o turismo rural

Martha Cecilia Vinasco Guzmán

Biofiltro con cascarilla de arroz y pasto vetiver (Chrysopogon zizanioides) para el tratamiento del efluente de la PTAR del INPEC - Yopal, Casanare, Colombia

Biofilter with rice husk and vetiver grass (Chrysopogon zizanioides) for the treatment of effluent from the WWTP of INPEC - Yopal, Casanare, Colombia

Biofiltro com casca de arroz e vetiver (Chrysopogon zizanioides) para

o tratamento de efluente da PTAR INPEC - Yopal, Casanare, Colômbia 
Biodegradación estimulada de los suelos contaminados con pesticidas organoclorados

Stimulated degradation of soils contaminated with

organochlorine pesticides

Biodegradação do solo estimulou contaminado com pesticidas

organoclorados

María Kopytko, Sandra Natalia Correa-Torres, Martha Jhoana Estévez- Gómez

Evaluación de la efectividad de distintas formulaciones de jabón con extracto de Hypericum mexicanum $\mathrm{L}$.

Evaluation of the effectiveness of different formulations

of soap with extract of Hypericum mexicanum $L$.

Avaliação da eficácia de diferentes formulações de sabão

com extracto de hipericão mexicanum L.

Diana Carolina Corzo-Barragán, Diana Milena Gaitán-Vaca

Efectos tóxicos del paracetamol en la salud humana y el ambiente

Toxic effects of paracetamol on human health and the environment

Efeitos tóxicos do paracetamol na saúde humana e no ambiente

Rosa Leonor Acevedo-Barrios, Carlos Alberto Severiche-Sierra, Jose Del Carmen

Jaimes Morales

Contaminación de suelos y aguas por hidrocarburos en Colombia. Análisis de la fitorremediación como estrategia biotecnológica de recuperación

Contamination of soil and water by hydrocarbons in Colombia. Analysis of phytoremediation as a biotechnology strategy for recovery

Solo e água contaminação por hidrocarbonetos em Colômbia. Análise de fitorremediação como estratégia de recuperação de biotecnologia

Johana Andrea Velásquez Arias

Sistema híbrido fotovoltaico (FV) con interacción a la red para zonas rurales de Colombia

(PV) photovoltaic hybrid system with interaction to the network to rural areas of Colombia

Sistema híbrido fotovoltaico (FV) com rede de interação para a Colômbia rural

Iván Mauricio Ostos Rojas, Carlos Andrés Collazos Morales,

Hermes Enrique Castellanos Acuña, Claudia Patricia Fernández Arévalo

169 


\section{DOCUMENTOS DE TRABAJO}

Actividad antimicrobiana de extractos etanólicos de propóleos obtenidos de abejas Apis mellifera

Antimicrobial activity of ethanol extracts of propolis obtained of bees Apis mellifera

Actividade antimicrobiana de extractos etanólicos de própolis obtido abelha Apis mellifera.

Análisis de un modelo agroforestal cafetero en el municipio de Valparaíso, Caquetá, Colombia.

Analysis of a coffee agroforestry model in the municipality of Valparaíso, Caquetá, Colombia.

Análise do modelo cafetero agro-florescente no município de

Valparaíso, Caquetá, Colômbia.

\section{Empleo del estropajo común (Luffa cylindrica) en la remoción} de contaminantes.

Use of the common sponge (Luffa cylindrica) in the removal of contaminants

Usando a bucha comum (Luffa cylindrica) na remoção

de contaminantes.

Ricardo Ignacio Pereira-Martínez, Juan Fernando Muñoz-Paredes,

Manejo de biosólidos y su posible aplicación al suelo, caso Colombia y Uruguay

Management of biosolids and its possible application to the soil, case Colombia and Uruguay

Gestão de biossólido e sua possível aplicação ao solo, caso Colômbia e Uruguai

Alexander Rodrigo Melo Cerón, Alejandra Rodríguez González, Juan Manuel González Guzmán 


\section{Comparación de la reglamentación para el manejo de lodos}

provenientes de agua residual en Argentina, Chile y Colombia

Comparison of the regulations for the management of sludge from waste water in Argentina, Chile and Colombia

Regulamentos de comparação para a manipulação de lamas de águas residuais na Argentina, Chile e Colômbia

Fabián Andrés Ospina López, Alejandra Rodríguez González, Juan Manuel González Guzmán

Análisis técnico, socioeconómico y ambiental de la electrificación con energía solar fotovoltaica aislada para vivienda rural en Hato Corozal, Casanare, Colombia

Technical analysis, socio-economic and environmental of the electrification with photovoltaics isolated for rural housing in Hato Corozal, Casanare, Colombia

Análise técnica, socioeconômica e ambiental da electrificação solar fotovoltaica para a habitação rural isolada em Hato Corozal, Casanare, Colombia

Edwin Blasnilo Rúa Ramírez, Andrea Isabel Barrera Siabato, Martín Gómez Orduz

Cómo citar los artículos publicados

en el Volumen 8 Número 1 enero - junio de 2017 


\section{Editorial}

Desde el primer número publicado en el año 2009, la Revista de Investigación Agraria y Ambiental RIAA, ha ido posicionando su proyecto editorial. RIAA es una revista científica de circulación semestral, que recibe artículos bien sea en español, inglés o portugués; en todos los casos los resúmenes de los mismos aparecen en tres idiomas, a saber, español, inglés y portugués. Próximamente, todos los artículos publicados contarán con versión completa en inglés con miras a una pronta inclusión en la base de datos bibliográfica Scopus y otros índices de citación.

La decidida vocación internacional de RIAA ha permitido su inclusión en bases de datos latinoamericanas e internacionales, 14 indexaciones le generan presencia en bases de datos, sistemas de indización, plataformas de evaluación de revistas, directorios selectivos, portales especializados y catálogos hemerográficos. Con ocho años de publicación ininterrumpida, a la fecha se tienen 209 artículos publicados, básicamente resultados de investigaciones científicas confiadas por académicos de todo el mundo.

La gestión editorial de los manuscritos postulados para publicación en la revista se realiza actualmente través de la Plataforma Open Journal System OJS, garantizando un riguroso y transparente sistema doble ciego de evaluación, una especializada corrección de estilo y una óptima diagramación e impresión. RIAA considera que autores y evaluadores son actores de buena fe en sus procesos editoriales, por lo cual estos declaran su compromiso ético y la no postulación simultánea en otras revistas o conflicto de intereses.

\footnotetext{
Reinaldo Giraldo Díaz

Docente Asociado

Escuela de Ciencias Agrícolas, Pecuarias

y del Medio Ambiente ECAPMA

Universidad Nacional Abierta y a Distancia UNAD
}

Los artículos de RIAA son publicados en formatos HTML y PDF y todos cuentan con el DOI respectivo, lo cual garantiza que se mantenga un alto nivel de visibilización mundial, el cual se irá complementando a futuro con otros sistemas de búsqueda como ORCID, PDFs dinámicos y ePUB, con conexión a gestores documentales como Mendeley, RefWorks, EndNote, y redes sociales científicas como academia.edu y ResearchGate.

RIAA ha manejado desde su inicio el doble ISSN: impreso y digital. En formato impreso se envían a los autores, los evaluadores y a los investigadores que así lo soliciten, hasta agotar existencias de los 1000 ejemplares que se imprimen en papel. Digitalmente, RIAA es accesible a texto completo, de forma gratuita, para la comunidad científica y académica de todo el mundo, siendo consultada tanto en HTML como en PDF.

El interés futuro de RIAA es posicionarse en el cuartil Q1 de Scopus y estar a la vanguardia de las revistas científicas del área agraria y ambiental latinoamericana. Actualmente en Google Scholar Metrics RIAA está catalogada como una de las mejores revistas colombianas en las temáticas agraria y ambiental (índice h5 de 8, en 2017, según Publish or Perish PoP). De esta manera, el equipo editorial de RIAA realiza un trabajo de avanzada que contribuye a mejorar el impacto de los investigadores y su producción científica.

\author{
Libia Esperanza Nieto Gómez \\ Docente Asistente \\ Escuela de Ciencias Agrícolas, Pecuarias \\ y del Medio Ambiente ECAPMA \\ Universidad Nacional Abierta y a Distancia UNAD
}




\section{Editorial}

From the first number published in the year 2009, the Revista de Investigación Agraria y Ambiental RIAA, has been positioning its editorial project. RIAA is a scientific journal of semiannually circulation, that receives articles either in Spanish, English or Portuguese; in all cases the summaries of them appear in three languages, namely English, Spanish and Portuguese. Soon, all articles published will have full version in English with a view to an early inclusion in the bibliographic database Scopus and other citation indexes.

The decided international vocation of RIAA has allowed its inclusion in databases Latin American and international, at present 14 indexations that generate it presence in databases, indexing systems, platforms of evaluation of journals, selective directories, specialized portals and newspaper catalogs. With eight years of uninterrupted publication, currently, RIAA have 209 articles published, basically results from scientific research entrusted by academics from around the world.

The editorial management of manuscripts postulates for publication in the journal is currently carried out through the Platform Open Journal System OJS, ensuring a rigorous and transparent system doubleblind evaluation, one specialized correction of style and an optimum layout and printing. RIAA considers that authors and evaluators are actors in good faith in their editorial processes, by which these declare their commitment to ethical and non-application simultaneously in other journals or conflict of interests.

Articles of RIAA are published in HTML and PDF formats and all have the DOI respective, which ensures that you maintain a high level of visibility on a global scale. which in future it will be complemented with other search systems as ORCID, PDFs, dynamic and ePUB, connected to document managers as Mendeley, RefWorks, EndNote, and social networks science as academia.edu and ResearchGate.

RIAA has handled from its beginning the double ISSN: printed and digital. In printed format the issues are sent to the authors, the evaluators and researchers who so request, until exhaustion of stocks of 1000 copies are printed on paper. Digitally, RIAA is accessible to full text, free of charge, to the scientific and academic community around the world, being consulted both in HTML as well as PDF.

The future interest of RIAA will be position it in the quartile Q1 of Scopus and be at the forefront of scientific journals of Latin American on agricultural and environmental areas. Currently in Google Scholar Metrics RIAA is ranked as one of the best magazines in Colombia into the thematic agrarian and environmental (index H5 8, in 2017, according to Publish or Perish PoP). In this way, the editorial team of RIAA performs a job of advanced that contributes to improve the impact of researchers and its scientific production.
Reinaldo Giraldo Díaz

Docente Asociado

Escuela de Ciencias Agrícolas, Pecuarias

y del Medio Ambiente ECAPMA

Universidad Nacional Abierta y a Distancia UNAD
Libia Esperanza Nieto Gómez

Docente Asistente

Escuela de Ciencias Agrícolas, Pecuarias

y del Medio Ambiente ECAPMA

Universidad Nacional Abierta y a Distancia UNAD 


\section{Editorial}

Desde o primeiro número publicado no ano 2009, a Revista de Pesquisa Agrária e Ambiental RIAA, têm ido posicionando o seu projeto editorial. RIAA é uma revista científica de circulação semestral que aceita artigos em espanhol, inglês ou português e em todos os casos os resumos desses artigos são mostrados nas três línguas. Proximamente, todos os artigos serão publicados completos em inglês visando uma indexação na base de dados bibliográfica Scopus assim como outros índices de citação.

A vocação internacional decidida do RIAA tem permitido sua indexação nas bases de dados de América Latina e internacionais. Até agora, 14 indexações mostram presença em bases de dados, sistemas de índices, plataformas de avaliação de revistas, diretórios seletivos, portais especializados e catálogos hemerográficos. Durante 8 anos de publicação continua, tem-se 209 artigos publicados, basicamente de resultados de pesquisas científica realizadas por académicos do mundo inteiro.

O procedimento editorial dos manuscritos candidatados para publicação no jornal é realizado pela plataforma Open Journal System OJS, garantindo o estrito e transparente sistema de dupla avaliação cega, correção de estilo especializado e ótima diagramação e impressão. RIAA considera que tanto autores quanto avaliadores são atores de boa fé nos seus processos editoriais, fato pelo qual declaram seu compromisso ético à não submissão simultânea em outros jornais ou conflito de interesse.
Os artigos do RIAA são publicados em formatos HTML e PDF e todos tem o respectivo DOI, garantindo um alto nível de visualização no mundo inteiro. No futuro, será complementado com outros sistemas de buscas como ORCID, PDFs dinâmicos e ePUB, com conexões aos gestores documentais como Mendeley, RefWorks, EndNote, e redes sociais científicas como academia. edu e ResearchGate.

RIAA tem administrado desde o começo a dupla ISSN: impresso e digital. O formato impresso enviase para autores, avaliadores e pesquisadores que solicitam este formato, até acabar os 1,000 exemplares impressos. No formato digital RIAA mostra o texto completo de forma gratuita para toda comunidade científica e académica ao redor do mundo e pode ser consultada em HTML e PDF.

O interesse mais próximo do RIAA é se colocar no quartil Q1 de Scopus e estar entre os melhores jornais científicos na área agronômica e ambiental de América Latina. $\mathrm{Na}$ atualidade, o Google Scholar Metrics tem o RIAA catalogado como um dos melhores jornais colombianos nos temas agrário e ambiental (índice h5 de 8, em 2017, según Publish or Perish PoP). Assim, a equipe editorial do RIAA faz um trabalho importante que contribui a melhorar o impacto dos pesquisadores y sua produção científica.

\author{
Reinaldo Giraldo Díaz \\ Docente Asociado \\ Escuela de Ciencias Agrícolas, Pecuarias \\ y del Medio Ambiente ECAPMA \\ Universidad Nacional Abierta y a Distancia UNAD
}

\author{
Libia Esperanza Nieto Gómez \\ Docente Asistente \\ Escuela de Ciencias Agrícolas, Pecuarias \\ y del Medio Ambiente ECAPMA \\ Universidad Nacional Abierta y a Distancia UNAD
}


